\title{
A study of the application of Brain Atlas with and without $+\mathrm{Gz}$ acceleration conditions
}

\author{
Yifeng $\mathrm{Li}^{\mathrm{a}}$, Lihui Zhang ${ }^{\mathrm{a}}$, Tao Zhang ${ }^{\mathrm{b}, *}$ and Baohui $\mathrm{Li}^{\mathrm{a}}$ \\ ${ }^{a}$ Air Force Institute of Aeromedicine, Beijing, China \\ ${ }^{\mathrm{b}}$ Department of Automation, School of Information Science and Technology, Tsinghua University, \\ Beijing, China
}

\begin{abstract}
The purposes of this study were to utilize Brain Atlas to investigate the fluctuations in the characteristics of human EEG, with and without $+\mathrm{Gz}$ acceleration produced by human centrifuge, and also to examine the $\mathrm{G}$ load endurance of human body. The Brain Atlas of the EEG signal with and without $+\mathrm{Gz}$ acceleration in a static state were compared in order to reveal the correlation and differences. When compared with those in a static state, it was found that for the EEG readings of the subjects undergoing $+\mathrm{Gz}$ acceleration conditions, the energy and gray scale values of the low-frequency component-delta rhythm showed significant increases, while the energy and gray scale values of the high-frequency component-beta rhythm showed significant decreases. Among these, the beta 2 rhythm was determined to be significantly inhibited. These fluctuations suggested that the ischemia conditions of brain had been improved. Also, the recoveries in the energy and gray-scale values were determined to be faster, which suggested that the G load endurance of human body had been enhanced. The Brain Atlas was found to show observable changes in color. The experimental results indicated that the Brain Atlas was able to provide assistance during the exploration of the fluctuations in the characteristics of EEG, and provided a criterion to assist in the observations of the function state fluctuations of human brain with $+\mathrm{Gz}$ acceleration. It also assisted in the evaluations of the $\mathrm{G}$ load endurance of human body.
\end{abstract}

Keywords: Brain Atlas, $+\mathrm{Gz}$, change characteristics

\section{Introduction}

In the field of aerospace, in order to prevent pilots experiencing air syncope during flight, the human physiological signals under $+\mathrm{Gz}$ acceleration conditions require monitoring and further research. In the fields of aviation and aerospace, the terms "overweigh" and "overload" are often used to explain the influence of inertia force. Several times of $G$ is equivalent to several times of gravity acceleration. Under $+\mathrm{Gz}$ acceleration conditions, the blood mass of the human body transfers from the head to the lower body under the action of inertia force. During this time, the brain can easily experience syncope due to ischemic hypoxia. Many types of physiological signals, including EEG, have been studied to obtain the early warning signs of syncope. Various of methods have been implemented, such as wavelet transformation [1,2]; artificial neural networks [3,4]; chaos and fractal methods [5]; and so on. Each of these methods has its own advantages and disadvantages. The method used most commonly is still spectrum analysis technology $[6,7]$. The EEG quantitative analyses which are based on spectrum analysis

\footnotetext{
${ }^{*}$ Corresponding author: Tao Zhang, Department of Automation, Tsinghua University, Beijing, China. E-mail: taozhang@ tsinghua.edu.cn.
}

0928-7329/17/\$35.00 (C) 2017 - IOS Press and the authors. All rights reserved

This article is published online with Open Access and distributed under the terms of the Creative Commons Attribution NonCommercial License (CC-BY-NC 4.0). 
are able to further improve the analysis level of the EEG. A Brain Atlas (BA) is also referred to as Brain Electrical Activity Mapping (BEAM), or Quantitative Electroencephalogram (qEEG). It is able to visually and clearly reflect the neural activity of the brain by using a color plane graph, and a side location graph of left, right, three other dimensions, or many dimension forms. A Brain Atlas is one of the most widely used methods for EEG quantitative analysis. It has the advantages of easily grasped operation technology, rapid analysis results, and an intuitive and eye-catching expression. Currently, it has undergone a great deal of clinical applications [8-13].

In this study, the EEG data under $+\mathrm{Gz}$ acceleration conditions were first obtained. Then, the change characteristics of the EEG under $+\mathrm{Gz}$ acceleration conditions, and without $+\mathrm{Gz}$ acceleration, were compared and discovered using the BA analysis method. In this study, this was the first time that EEG data from a human body undergoing $+\mathrm{Gz}$ acceleration were obtained using human centrifuge. This was also the first time BA technology had been applied to an EEG study regarding $+\mathrm{Gz}$ acceleration. The result showed that the BA analysis was able to intuitively and more affectively assist in obtaining the EEG characteristics. Also, the BA was found to be an effective index by which to observe EEG changes both with and without $+\mathrm{Gz}$ acceleration.

\section{Data acquisition and experimental process}

The main equipment of this experimental study was a new human centrifuge. Five healthy volunteers were selected as the experimental subjects. The subjects experienced $+\mathrm{Gz}$ exposures according to the curve set by the centrifuge. The set curve was as follows: beginning from $1 \mathrm{G}$, a maximum $\mathrm{G}$ value with a certain growth rate was reached, which was continued for 10 to 15 seconds before finally returning to $1 \mathrm{G}$ with a certain growth rate once again. The max $\mathrm{G}$ value began from $2.5 \mathrm{G}$, and increased by $0.5 \mathrm{G} / \mathrm{r}$ until the subjects reached their endurance endpoints or a stop index appeared. The doctors monitored the subjects' ear pulse in real-time, as well as their ECG and other physiological signals, and comprehensively judged the endurance end of subjects according to the level situation of their ear pulse, combined with the subjective description of the perimeter lamps disappearing or the central lamp becoming fuzzy. This was also combined with the expressions of subjects. The EEG electrodes of 16 leads were installed in accordance with their positions in a 10-20 system of international EEG standard electrodes installation method. The electrode at the ipsilateral earlobe (A1 or A2) was taken as the reference electrode for all the electrodes [14]. The obtained data was filtered and displayed by the supporting software of the EEG physiological parameters recorder produced by Shanghai NuoCheng Company. Moreover, the data underwent frequency spectrum analysis, and mapping BA, as well as other corresponding functions.

\section{Results}

The EEG data of 16 leads first passed the pretreatment, which included a low pass digital filtering below $30 \mathrm{~Hz}$, then a fast Fourier transformation at the sampling frequency of $128 \mathrm{~Hz}$ (FFT transformation) from the time domain transforming to the frequency domain and period diagram of each segment were obtained. For each subject, the data of 10 seconds during the $+\mathrm{Gz}$ action, and the data of 10 seconds before the $+\mathrm{Gz}$ action (at a quiet state) were selected. Then, each of the BA of the alpha, beta, delta, and theta rhythms was completed. The BA included top views, left views, and right views, in order to better observe the energy changes of each rhythm at different locations in the brain. In this study, the segmentation criterion was as follows: delta $=0$ to $3.5 \mathrm{~Hz}$; theta $=4$ to $7.5 \mathrm{~Hz}$; alpha $1=8$ to $11 \mathrm{~Hz}$; 


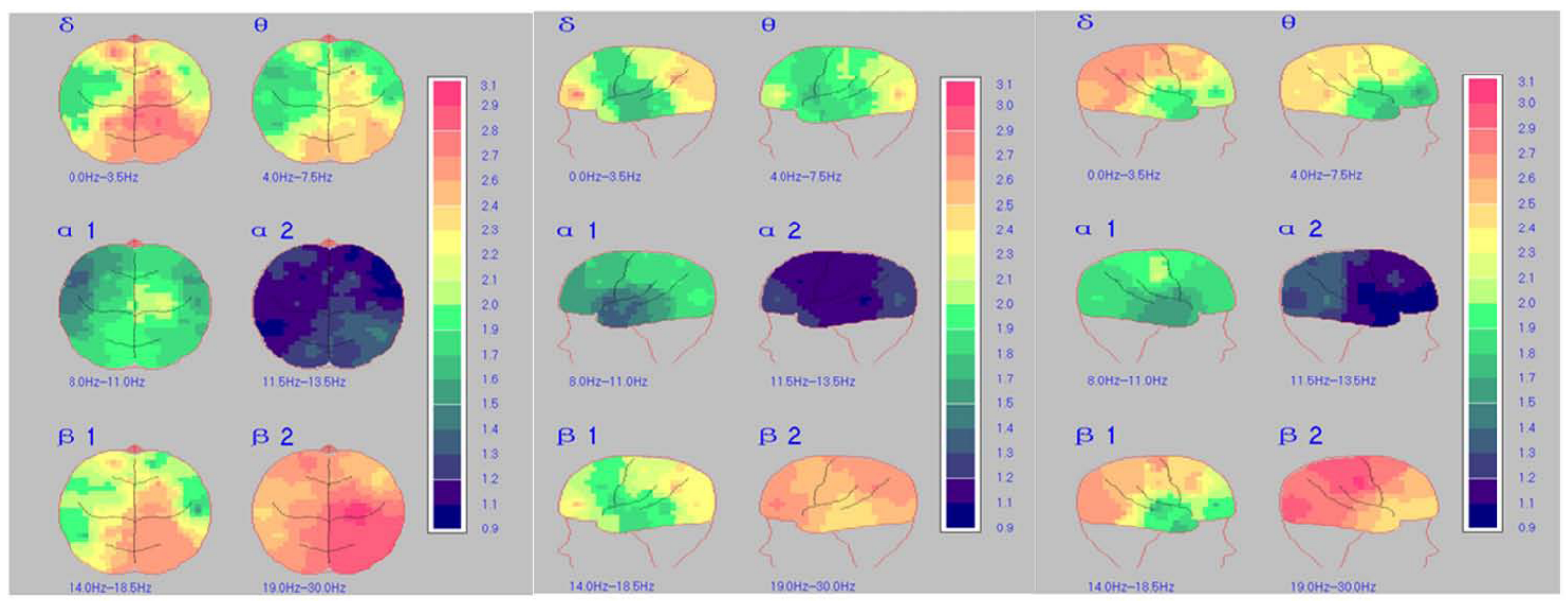

Fig. 1. BA distribution of subject when at static state, that is when $1 \mathrm{G}$.

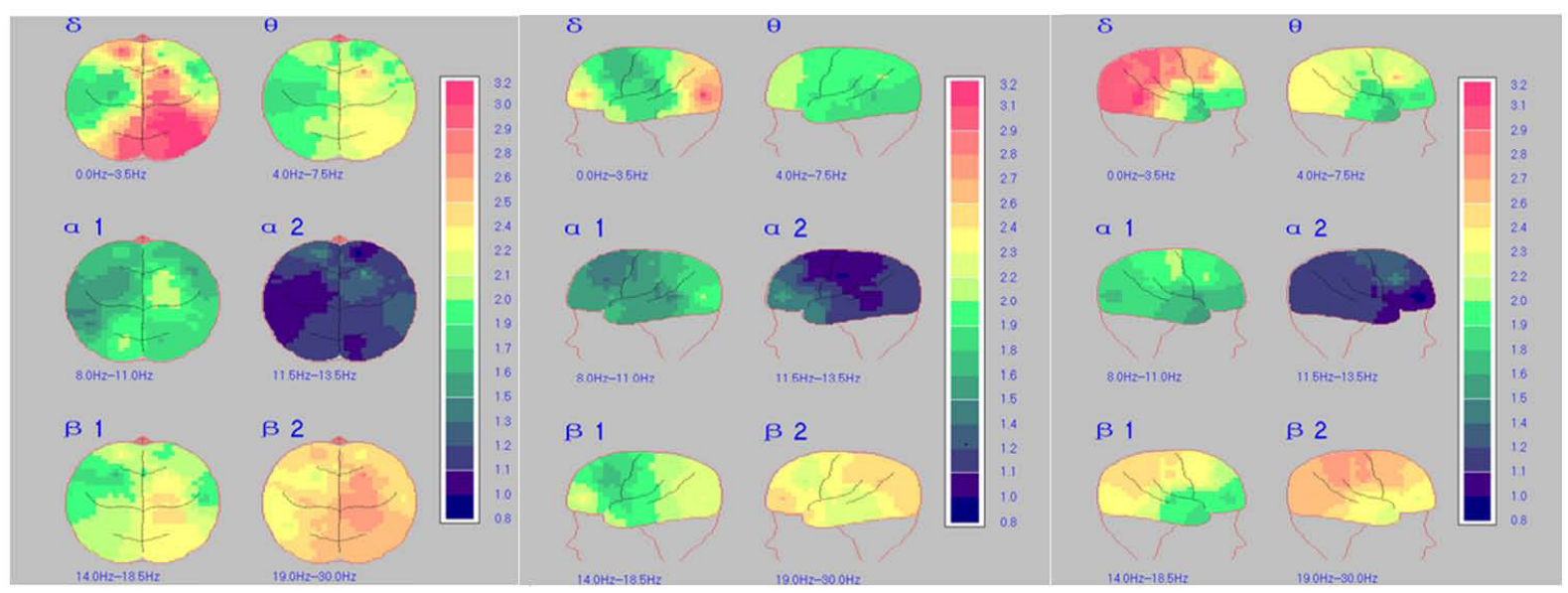

Fig. 2. BA distribution of subject when $3 \mathrm{G}$.

alpha $2=11.5$ to $13.5 \mathrm{~Hz}$; beta $1=14$ to $18.5 \mathrm{~Hz}$; and beta $2=19$ to $30 \mathrm{~Hz}$. The BAs of the six types of rhythms were observed. Figure 1 shows the BA for a subject at a static state which was under $1 \mathrm{G}$ of acceleration. Figure 2 is the BA for a subject undergoing a $3 \mathrm{G}$ acceleration. The figure shows the top view, left view, and right view of the EEG, respectively. The scale represents the energy value. In the figure, the different colors indicate the various energy distributions.

The BAs of the five subjects at a static state, and also undergoing $+\mathrm{Gz}$ acceleration, all showed significant changes. Among these, the energy value of the alpha frequency band decreased slightly. The delta and beta frequency bands were the most obviously manifested. From left and right views, it can be seen that the delta rhythm had very high energy at the right rear of the brain (the right top part), right occipital part, right posterior temporal part, and occipital midline point part, as well as the right central part. The energy was also relatively high at the left occipital part, and the left top part. Moreover, the energy increased significantly, and the gray scale value of the color rose significantly. For example, it was found to rise approximately five energy grades, with the local value closing to the highest value. Meanwhile, the energy of the beta frequency band in the entire brain showed obvious decreases. The beta rhythm 
displayed high energy at the right rear of brain (right top part), right occipital part, right posterior temporal part, occipital midline point part, and top midline point part, as well as the right central part. The energy was found to also be relatively high at the left occipital part, and the left top part. Moreover, the energy decreased significantly, and the gray scale value of the colors declined significantly. In particular, the decreasing of the beta2 frequency band was found to be the most obvious. The energy of the beta2 frequency band decreased sharply, and the gray scale value of the colors showed an obvious decline of approximately six energy grades, which were found to manifest more obviously in right view figure.

\section{Results analysis}

In this study, the BAs of the subjects when in a static state, as well as under $+\mathrm{Gz}$ acceleration conditions, showed obvious changes. It was determined that the energy of the delta frequency band increased, and the energy of beta frequency band decreased. This could be explained by the fact that the ischemia degrees of the subjects' bodies were increasing, sober consciousness was lost, and the inhibition degrees of the brain were increasing. This was related to the blood was transferring from the head to the lower parts of the subjects' bodies. Therefore, the cerebral areas underwent ischemia and hypoxia under the $+\mathrm{Gz}$ acceleration conditions, and as a result of the centrifugal force. It was found that, having been effected by the blood and other body circulations, the subjects' ischemia attraction to the right side of body was larger than that of the left side of body. The ischemia degrees of the right side of body became serious, and displayed a close relationship with this special body position. In addition, the beta rhythms, especially the beta 2 rhythms, changed significantly. In a human lower body negative pressure experiment in 2000, it was observed that a remarkable phenomenon occurred in which the power percentage index of the beta 2 frequency band $(20 \sim 29.8 \mathrm{~Hz})$ displayed a dumping one minute before the human body experienced syncope. These findings suggested that further investigations of the change characteristics of the power spectrum of the beta band in EEG could potentially assist in a deeper understanding of the changes in the brain function state. These changes cannot be understood only by judging the diagrams or identifications by the naked eye prior to G-LOC or syncope [15]. In this study, the changes in the BA further verified this one point. Therefore, the beta2 rhythm may become the symbol rhythm of the change characteristics of EEG under $+\mathrm{Gz}$ acceleration conditions in the future. This rhythm has important meaning for the future examinations of the function states of the brain using EEG.

The changes in the brain function state are judged and evaluated mainly through changes in the energy values, along with changes in the color gray values of the delta and beta frequency bands in the BA. Under $+\mathrm{Gz}$ acceleration and centrifugal force conditions, a large amount of blood transfers from the head to the low parts of the body. This can easily lead to brain ischemia and ischemic hypoxia, and may cause the human body to exhibit syncope. Therefore, the brain appears also to have a series of functional changes. The energy values, along with the gray scale values of the delta rhythm tend to rise, which suggests that the injury states of the brain cell functions are worsening, and that the brain functions have become damaged. The decline in the energy and gray scale values suggest that the damaged brain cell functions are now recovering. When the power and gray scale values decline more quickly, this indicates that the recovery of the brain cell functions was faster following the $\mathrm{G}$ load action. In other words, the recovery of the brain functions was faster, which indicated that the human endurance in resisting the G load was stronger. When the energy and gray scale values of the beta rhythm declined, this suggested that the blood supply to the brain was insufficient, and the brain as in an ischemia state. Also, the conscious degree of the brain was decreasing. In the cases where the power and the gray scale values rose, this indicated that that blood supply to the brain was improving, and also that the 
brain ischemia state and brain circulation were improving. The power and gray scale values rising more quickly suggested that the recovery of the brain blood supply was both faster and more obvious after the $\mathrm{G}$ load action, and indicated that the human endurance in resisting the $\mathrm{G}$ load was stronger. Since the differences between the energy values and energy grades for the different individuals were large, it was impossible to formulate a unified judgment or evaluation criterion for the energy values of BA in this current study. However, the changes in the brain function state could be observed through Significant Probability Mapping (SPM), which was the BA displaying the differences between the subjects' data and normal human data by a statistical treatment, and using SD as the unit expression. The deviation degrees of the SPM transiting from high to low suggested that the brain cell functions were recovering normally. The deviation degree changes from slower and faster suggested that the recovery of brain functions were more significant and faster, and that the $\mathrm{G}$ endurance was improved. It was considered as to whether certain standards could be made for judgments regarding when the human body was near syncope, as well as evaluating the $\mathrm{G}$ endurance of the human body according to large and small values of the deviation degrees. These topics may become the focus in future research directions.

In summary, the BA is an important assistant means of frequency spectrum analysis, as well as other digital analyses of EEG. It was found to be an effective index to assist in the observations of the changes in brain function states under $+\mathrm{Gz}$ acceleration conditions, and also to evaluate the $\mathrm{G}$ endurance of the human body. It is expected to become the future physiological index for evaluating the function states of the human brain, along with the human body's $\mathrm{G}$ endurance.

\section{Conclusions}

In this study, human EEG data before and after $+\mathrm{Gz}$ action were obtained using a new type of human centrifuge. The BA of the EEG signals under $+\mathrm{Gz}$ acceleration conditions and before $+\mathrm{Gz}$ actions when the body was at a static state were compared. It was found that the BA displayed obvious color changes, and the brain rhythms also change significantly, which mainly showed that the energy of the low frequency delta rhythms increased significantly, and the energy of the high frequency beta rhythm decreased significantly. In particular, the beta2 rhythms were found to change more prominently. The energy values and corresponding gray scale values of the delta frequency bands declined. Meanwhile, the energy values and corresponding gray scale values of the beta frequency band rose. These findings indicated that the blood supply to the brain was improving, as were the brain's ischemia state and circulation. When the power and gray scale values rose more quickly, this indicated that the brain's blood supply recovery was faster and more obvious after the G load action, and that the human endurance in resisting the $\mathrm{G}$ load was stronger. In the BA of this study, the energy of the beta2 rhythm changes was obvious, which verified the viewpoints of previous related studies. In summary, the BA showed an ability to assist in the understanding of the changes in brain function states more intuitively and comprehensively, and provided an effective index in the observations of the changes of brain function states under $+\mathrm{Gz}$ acceleration conditions, as well as the evaluations of the $\mathrm{G}$ endurance of the human body. Therefore, the BA has a practical significance and important application value in helping to understand the situations of brain circulation and changes in brain function states, along with improving the understanding of the signs in a subject undergoing $+\mathrm{Gz}$ acceleration during actual training situations.

\section{Conflict of interest}

None to report. 


\section{References}

[1] Pan JS, Yue L. A ridge extraction algorithm based on partial differential equations of the wavelet transform. In: 2014 IEEE Symposium on Computational Intelligence for Multimedia, Signal and Vision Processing (CIMSIVP); 2014.

[2] Kumar K, Mustafa N, Li JP, Shaikh RA, Khan SA, Khan A. Image edge detection scheme using wavelet transform. In: 2014: 11th International Computer Conference on Wavelet Active Media Technology and Information Processing (ICCWAMTIP); 2014.

[3] Jayakumar S, Kanna R. Inspection System for Detecting Defects in a Transistor using Artificial Neural Network (ANN). In: Proceedings of the International Conference on Communication and Computational Intelligence 2010 (PICCCI), 2010.

[4] Borenovic M, Neskovic A. ANN Based Models for Positioning in Indoor WLAN Environments. In:19th Telecommunications Forum TELFOR 2011; 2011.

[5] Li P, Wang GH, Yan XP, Gong P, Shi WB, Song CY. Chaos characteristics analysis of transient signals for phaselocked synthesizer based FHC system. In: 2014 14th International Symposium on Communications and Information Technologies (ISCIT); 2014.

[6] Lv WT, Shen C, Gui F, Tian ZS, Jiang DZ. Real-Time Spectrum Analyzer Based on All Phase FFT Spectrum Analysis. In: 2013; Fourth International Conference on Digital Manufacturing and Automation (ICDMA); 2013.

[7] Li H, Liu YD, Lu JH, Zheng T, Yu XH. Suppressing EMI in Power Converters via Chaotic SPWM Control Based on Spectrum Analysis Approach, IEEE Transactions on Industrial Electronics 2014; 61(11): 6128-6137.

[8] Melo MC, Del Claro TTS, de Sa AAR, Soares AB, Andrade AO, Linhares ND. Proposal of a three-dimensional brain mapping system based on the quantitative analysis of the electroencephalographic signal. In: 5th ISSNIP-IEEE Biosignals and Biorobotics Conference (2014); Biosignals and Robotics for Better and Safer Living (BRC); 2014.

[9] Sun ZJ, Wang G, Li K, Zhang ZL, Bao G. Cerebral functional connectivity analysis based on scalp EEG in epilepsy patients. In: 2014 7th International Conference on Biomedical Engineering and Informatics (BMEI); 2014.

[10] Prueckl R, Kapeller C, Potes C, Korostenskaja M, Schalk G, Lee KH, Guger C. cortiQ-Clinical software for electrocorticographic real-time functional mapping of the eloquent cortex. In: 2013 35th Annual International Conference of the IEEE Engineering in Medicine and Biology Society (EMBC); 2013.

[11] Najumnissa D, Shenbagadevi S. Localisation of Epileptic Seizures by-A Novel Technique Topographical Brain Electric Activity Mapping. In: ICBPE 2006 International Conference on Biomedical and Pharmaceutical Engineering, 2006.

[12] Kawada M, Leahy RM. Electrical Brain Mapping of Motor Imagination Using the Minimum Norm Solution. In: International Symposium on Communications and Information Technologies, 2006; ISCIT '06; 2006.

[13] Wang L, Xu GZ, Yang S, Song Y, Wei YF, Yan WL. Research on Event Related Potential Elicited by Number Recognizing and Arithmetic Calculating. In: Joint Meeting of the 6th International Symposium on Noninvasive Functional Source Imaging of the Brain and Heart and the International Conference on Functional Biomedical Imaging, NFSI-ICFBI 2007; 2007.

[14] Nakamura M, Chen Q, Sugi T, Ikeda A, Shibasaki H. Technical quality evaluation of EEG recording based on electroencephalographers' knowledge. Medical Engineering \& Physics 2005; (27): 93-100.

[15] Chen YS, Jiao ZG, Yan GD, Jin ZR. The characteristics of cerebral functional indices during occurrence of presyncopal symptom induced by lower body negative pressure. Chinese Journal of Aerospace Medicine 2000; 11(3): 145-148. 